\title{
Hypertension, diabetes, obesity and other ailments in contemporary Brazil
}

Since the 1960s, Brazil has seen a change in its morbidity and mortality profile, moving from a situation where there was the prevalence and relevance of infectious diseases and entering an ascending curve of both deaths and morbidities due to long-term noninfectious clinical pathologies, often marked by irreversibility. This scenario is also characterized by an increase in deaths from violence and accidents, and lesions and injuries resulting from so-called "external causes."

This new panorama of the Brazilian population has two sides. The most positive aspect is that thanks to the major vaccination campaigns, the universalization of primary health care, improvements in living conditions and the slow but vital process of ensuring basic sanitation, the country has eliminated some infectious diseases, such as poliomyelitis, smallpox and measles. However, others persist and require major investment to keep them in check, such as AIDS (which for many is becoming a chronic disease), dengue, tuberculosis, malaria and Hansen's disease (leprosy). The most negative aspect of the Brazilian health situation is the presence, persistence, and in some cases, the reemergence of non-communicable diseases such as diabetes, obesity and hypertension, which are the main focus of this issue of Ciência \& Saúde Coletiva.

There is a mirror-effect between the morbidity and mortality status and current living situations and conditions. Most diseases are associated with lifestyle: the quality of nutrition, a sedentary lifestyle, stress, working conditions, environmental pollution and social violence. And, equally important, the new profile takes into account the aging of the population - the most important phenomenon of contemporary Brazilian demographics - which is simultaneously a bonus for the country and a cause of concern for the health sector. It is clear that aging is not and cannot be considered a disease. However, when people do not take care of themselves during their youth and adult lives - genetic problems aside - in old age they tend to be counted among the number of those suffering from chronic and degenerative processes. It should be stressed that such ailments are not peculiar to the elderly, although they are its main victims: hypertension; diabetes; obesity; cardiovascular diseases; neoplasms; respiratory diseases; kidney disease; musculoskeletal diseases; mental health problems; and diseases of the sensory, dental and periodontal organs.

There are three characteristics common to non-communicable health problems, including the scourge of violence and accidents. Firstly, their multiple causality do not permit a single course of action for which health professionals are better prepared. This effectively raises the question of the adequacy of their training to suit the new profile of morbidity and mortality in the country. Secondly, any action requires the involvement of the individuals and their accountability for their state of health. In other words, they can and should do more both personally and socially - without waiting for the doctor's advice - preventing the complications and the factors that cause the new ailments mentioned above. Thirdly, healthy aging depends on the history and lifestyle of the individuals, their environment and their integration in social life while they are still young.

In conclusion, due to the current health situation of Brazilians, the medical and public health authorities are increasingly being called upon to assume their role as a hybrid science that involves biological, psychic and social expertise. As Marcel Mauss put it, both health and illness constitute a "total social fact": when the body is in pain, the mind feels it and social life is jeopardized. On the contrary, when one is healthy, the silence of the body and the joy of the soul prevail.

Maria Cecília de Souza Minayo

Editor-in-Chief 